\title{
On-Line Optimizing Control of a Simulated Continuous Yeast Fermentation
}

\author{
Andersen, Maria Y.; Asferg, L.; Brabrand, H.; Karim, N.; Jørgensen, Sten Bay
}

Published in:

American Control Conference

Publication date:

1989

Document Version

Publisher's PDF, also known as Version of record

Link back to DTU Orbit

Citation $(A P A)$ :

Andersen, M. Y., Asferg, L., Brabrand, H., Karim, N., \& Jørgensen, S. B. (1989). On-Line Optimizing Control of a Simulated Continuous Yeast Fermentation. In American Control Conference (pp. 2000-2005). IEEE.

\section{General rights}

Copyright and moral rights for the publications made accessible in the public portal are retained by the authors and/or other copyright owners and it is a condition of accessing publications that users recognise and abide by the legal requirements associated with these rights.

- Users may download and print one copy of any publication from the public portal for the purpose of private study or research.

- You may not further distribute the material or use it for any profit-making activity or commercial gain

- You may freely distribute the URL identifying the publication in the public portal

If you believe that this document breaches copyright please contact us providing details, and we will remove access to the work immediately and investigate your claim. 
ON-IINE OPTIEIZING COWHROL OF A SIHOLATED CONITIJOOUS YEAST FERABHTATION

\author{
Naria Y.Andersen, I.Asferg, H.Brabrand, N.Karin*, S. Bay Jorgensen \\ Instituttet for Keniternik, \\ Technical University of Denmark, Lyngby Denmark \\ $\star$ \\ Dept. of Agriculture and Chemical Engineering, \\ Colorado state University, Fort Collins, Colorado
}

\begin{abstract}
On-line optimizing control of a simulated fermentation is investigated using a non-segregated dynanic model of aerobic glucose linited growth of saccharomyces cerevisiae. The optimization procedure is carried out with an underlying adaptive regulator to stabilize the culture. This stabilization is especialIy important during the setpoint changes specified by the optinizing routine. A Iinear ARMAx model structure is used for the fermentation process with dilution rate as input and biomass as output variable. The parameters of the linear model structure are estinated using a pseudo linear regression method with bandpass filtering of in- and output variables in order to ensure low frequency validity of the estimated nodel. An IQ-regulator is used with iterative solution of the Riccati equation.
\end{abstract}

Simulation results illustrate the tuning of the underlying regulator, and the effect of perturbing the manipulated variable with a dither signal. The results demonstrate that an optimum can be reached smoothly in what corresponds to about twice the effective open loop response time. The results demonstrate the effectiveness of the stabilizing regulator in rejecting relatively short duration disturbances.

\section{INTRODUCTION}

optimization of fermentation processes is often carried out on continuous fermentors, where the effect of changes in parameters of the objective function may be investigated one at a time or in factorial experiments. In both types of experiments it is essential to wait for new steady state conditions before one can insert the next set of operating conditions. During on-line optimizing control an objective function is maximized on-line by manipulating one or more operating parameters without being forced to wait for steady state conditions between experiments. Thus on-line optmizing control is potentially a very time saving method.
The subject of optinizing control has been studied mainly during the last decade, and has been reviewed by Arkun and Stephanopoulus (1980). Bamberger and Iserman (1978) introduced the idea of performing estimation of a dynamic model and using the static information in the optimization. The basic approach of this paper is similar but includes also a regulator for disturbance rejection, and for tracking of the setpoint calculated by the optimizing level. sinilar regulators were applied by Garcia and Morari (1981) and Lee and Lee (1985). When nonlinear processes are being optimized then the desired regulator tuning may vary considerably with the present operating point, thus either a robust or an adaptive regulator may advantageously be applied.

Optimizing control of continuous ferwentation processes has been studied by Rolf and Lim (1984, 1985 and 1988) on a relatively complex simulation nodel (Lievense 1984), and on an experimental fermentor. Harmon et al. (1985 and 1987) uses a simpler simulation model, as does Golden and Yastie (1987).

In the present paper the tuning of a siso optimizing algorithm on a simulated fermentation process is studied by using filtering and a variable input perturbation. The optimization is initiated on either side of the optimal dilution rate in order to investigate the performance of the optimization under these very different dynamic conditions. Furthermore the behaviour towards different types of disturbances is investigated.

\section{OPTIMIZATION CRITERION AND PROCESS}

A simple optinization formulation for a biomass producing continuous fermentation is to maximize an objective function of the following form:

$$
\underset{D}{\operatorname{Max}} J=D X-C_{0}
$$


subject to:

$$
\begin{aligned}
& D_{\min }<D<D_{\max } \\
& y_{\mathbf{s}}=G u_{s}+d
\end{aligned}
$$

where $y$ is a scaled biomass $x$ and $u$ a scaled dilution rate $D$. clearly the scaling of the input and output only becomes significant when these varlables are vectors. The optimization problem may be solved for the (steady state) optimum u using eq. (2b) to substitute the biomags in eq. (1). In practice several more constraints may apply, and the limits of the constraints may be variable, e.g. due to the limited oxygen uptake capacity where an increase in $S$ would demand a decrease in the obtaina? ble $D_{\max }$.

The yeast fermentation process is modelled using an eigth order model developed by Lievense (1984). The model is able to describe the crabtree effect of yeast metabolism. The steady state gain dependence of dilution rate is shown in figure $1 \mathrm{a}$, and the objective function dependence of the dilution rate is shown in figure 1b. Clearly the steady state gain drops to very low values for dilution rates between 6 and 128 higher than the optimum value. The biomass is assumed measured measured every $6 \mathrm{~min}=\mathrm{T}$.

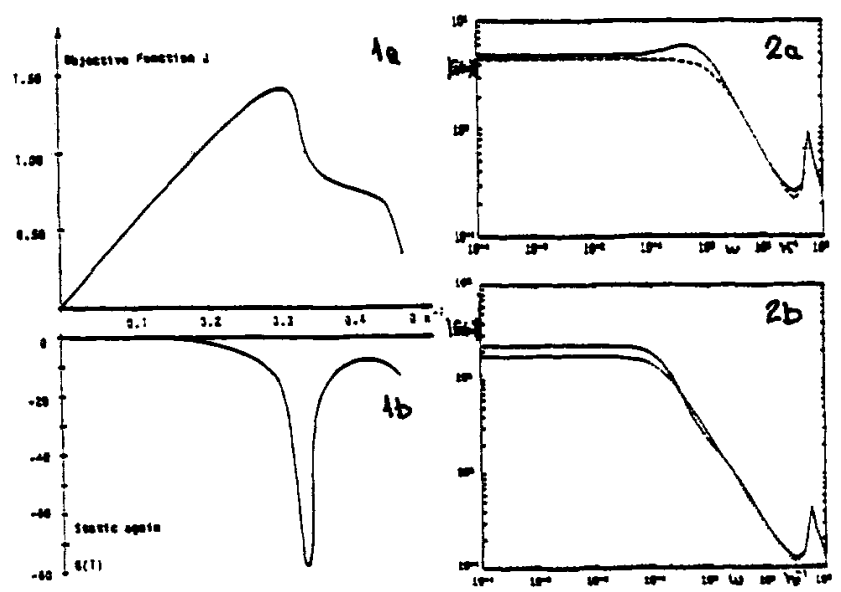

Figure 1: Dilution rate dependence, for $S_{0}=10 \mathrm{~g} / 1$, of:

a) the objective function, and b) the static gain.

Figure 2: Amplitude frequency function for linearized model (full line), and for estimated ARMAX model with $n_{n}=2$, $n_{b}=n_{c}=1$ (broken line). a) $D=\equiv_{1} 0.25 h^{2}=1$, $w^{b} \cong 0.1 h^{-1}$, and $w_{10}=10 h^{-1}$. b ${ }^{w}{ }_{D}=0.1 h^{\prime}$ and $w_{c l p}=10 h^{-1} i_{c h p}=1.0 h^{-1}$, and $w_{c l p}=20 \cdot h^{-1}$.

Optimization is attempted starting from both ends of the range of allowed dilution rates. The locally 1 inear dynamics are illustrated by the amplitude frequency functions shown in fig. $2 a-b$ for biomass over dilution rate. Note the low frequency resonnance peak at the lower dilution rate, this effect is even more pronounced at lower dilution rates. Note also that the corner frequency decreases with increasing dilution rate, for the two cases shown.

\section{METHODS}

The optimization control hierachy is illustrated in figure 3 (last page). The hierachy contains three levels. Since both the upper and lower levels depend upon the middle, this middle level is described first. At the middle level the parameters of an ARMAX model for the fermentation process are estimated:

$$
\begin{aligned}
& A\left(q^{-1}\right) y(t)=B\left(q^{-1}\right) u(t)+c\left(q^{-1}\right) e(t) \\
& A\left(q^{-1}\right)=1+a_{1} q^{-1}+\ldots+a_{n a} q^{-n a} \\
& B\left(q^{-1}\right)=b_{1} q^{-1}+\ldots+b_{n b} q^{-n b} \\
& C\left(q^{-1}\right)=1+c_{1} q^{-1}+\ldots+c_{n c} q^{-n c}
\end{aligned}
$$

Bandpass filtered values of process input and output are used for the estimation. The lowpass filter $L P$ is a fourth order Butterworth filter, and the highpass filter HP has the simple first order transfer function:

$$
\frac{1-z^{-1}}{1-k z}
$$

It is quite important to use filters in order to reduce bias in the parameter estimation and thereby obtain reliable estimation of the process dynamics (Ljung 1987) over the desired relatively wide frequency range. Reliable estimates of low frequency dynamics are needed when performing optimizing control, and similarly for the higher frequencies in order to perform satisfactory stabilizing control at the lowest control level. Amplitude functions obtained for one ARMAX model in figure $2 a-b$ is seen to represent the linear process dynamics reasonably well, but note that different filters were necessary at the two operating points. The parameter bias frequency distribution depends, as shown by Ljung (1987), upon the experimental design parameters $(\mathcal{D})$, such as: The filters, the sampling interval and the perturbation signal. Assume that the actual system can be described by the linear model:

$$
\begin{aligned}
y(t) & =G_{0}(q) u(t)+H_{0}(q) e(t) \\
& =G_{0}\left(e^{i w T}\right) u(t)+H_{0}\left(e^{i w T}\right) e(t)
\end{aligned}
$$

Where $G$ and $H$ are transfer functions, $i$ the complex operator, w the angular frequency, and $e(t)$ discrete time random noise. Estimating the parameters of a modelstructure, while prefiltering both the in- and outputs with the filter $L(q)$, then: 
$y(t)=G_{1}\left(e^{i W T}, \theta\right) u(t)+H_{1}\left(\left(e^{i w T}, \theta\right) e(t)\right.$

where $\theta$ is the parameter vector, and $G, H$ the identified transfer functions. If the number of data used is $N$, then, as the number of data goes towards infinity, the parameters will converge for a one step pgediction method towards the estimates $\theta$

$$
\begin{aligned}
& \theta^{*}=\arg \operatorname{nin} \int_{-w / T}^{\pi / T} G_{0}\left(e^{i w T}\right)-G_{1}\left(e^{i w T} y^{2} Q\left(w, \theta^{*}\right) d w\right. \\
& Q(w, \theta)=\frac{I_{u}(w)\left|L\left(e^{i w r}\right)\right|^{2}}{\left|H_{1}\left(e^{i w T}\right)\right|^{2}}
\end{aligned}
$$

$f_{u}(w)$ is the spectral density of the ifput signal. Eq. (6) expresses the weighting of different frequencies upon the converged parameter estimates. Thus this frequency weighting may be influenced by the choice of input signal and the filtering. In this investigation a PRBS signal is applied, which has a nearly flat spectrum, thus only the perturbation amplitude and the filtering affect the frequency range of the parameter estimation.

The parameter estimation is performed using a one step RELS with a variable forgetting factor, which is allowed to vary between 0.8 and 1.0 , using the method of Ydstie (1982). The method uses also Biermans (1977) factorization of the covariance matrix. An important tuning parameter for the variable forgetting factor algorith is product of the estimation horizon length $N_{h}$ and the expected measurement variance. This product $W$ must be selected such that the parametssq estimation is able to reduce the forgetting factor and thus allow new information, when available, to affect the estimates. When no new information is available, ie. the a priori prediction error is zero then no forgetting of old data takes place.

At the lowest control level stabilizing control is carried out, by means of a feedback loop. This loop is designed with a IQ controller, which minimizes the objective function:

$$
\begin{aligned}
& J_{\text {stab }}=z^{T}(t+N) Q_{y} z(t+N)+ \\
& \sum_{i=1}^{N-1} z^{T}(t+i) Q_{y} z(t+i)+u^{T}(t+i) Q_{u} u(t+i)
\end{aligned}
$$

with $z^{T}(t)=(y(t)-y(t-1), y(t-1)-y(t-2), \ldots$

$$
\begin{aligned}
& y\left(t-n_{a}+1\right)-y\left(t-n_{a}\right), u(t-1)-u_{\text {ref }} . . \\
& \left.u\left(t-n_{b}\right)-u_{\text {ref }}\right) .
\end{aligned}
$$

The effect of using incremental outputs is to allow low frequency variation of the output, whereas the low frequency behavior of the input is completely determined by $u$. Thus this controller is able to rejege medium and high frequency disturbances.

A nonminimal state representation is used for the low level controller design:

$z(t+1)=A 2(t)+B u(t)$

where, for $n_{a}=n_{b}=3$, the natrices are:

$$
a_{1} a_{2} a_{3} b_{2}-b_{1} b_{3}-b_{2}-b_{3} \quad b_{1}
$$

$A=\left[\begin{array}{llllll}1 & 0 & 0 & 0 & 0 & 0 \\ 0 & 1 & 0 & 0 & 0 & 0 \\ 0 & 0 & 0 & 0 & 0 & 0 \\ 0 & 0 & 0 & 1 & 0 & 0 \\ 0 & 0 & 0 & 0 & 1 & 0\end{array}\right] \quad B=\left[\begin{array}{l}0 \\ 0 \\ 1 \\ 0 \\ 0\end{array}\right]$

Further details of estiantion and control calculations are given in Brabrand and Jorgensen (1988).

The optinization is carried out at the the upper level. The steady state relationship eq. (2a), which is used as a constraint for the optinization, is derived from the ARMax model in eq. (3):

$$
\begin{aligned}
y_{g}=G(1) u_{s}+d \\
\text { where } G(1)=A^{-1}(1) B(1) \\
d=y_{w}-G(1) u_{w}
\end{aligned}
$$

As working point $y_{w}, u_{\text {, the lowpass }}$ filtered values of the dctual in- and outputs are used in deternination of an estimate of the constant term d. The gain estinate may become uncertain if the process gain 18 very low, therefore the static gain estimate is imited to: $-100<G(1)<0$

If $G(1)>0$ then the lower limit is used.

The optimization problen, eq.'s ( 1 and 2b) is solved explicitly. The determined optinal value is constrained to:

$$
0.0<a_{\text {opt }}<0.6
$$

The maximally allowed step length is inited toul The desired new value for the dilution rate is the reference value, $u$, for the input of the stabilizing ref'gulator. This value is constrained according to eq. 2a. The optimal solution is evaluated every $T_{\text {opt }} h$.

Since the optimization is based upon the static gain and the constant term $d$, the accuracy of these estimates is important. The process may be far from the optimum curing the initial phase of the optimization, or wen the behaviour of the fermentation or of the inputs has 
shifted. Thus in order to obtain reasonably accurate parameter estimates the perturbation level is allowed to vary according to the difference between the calculated optimal value a and the actual $u$. This difference is ogtnstrained as follows:

$$
0.1<1 a_{\text {opt }}-u_{w} 12.85<1.0
$$

The perturbation amplitude is set to a value defined by this constrained fraction multiplied by the maximal pertubation amplitude $a_{\max }$.

\section{RESULTS AND DISCUSSION}

The procedure followed in performing optimizing control on a simulated continuous yeast fermentation is as follows.
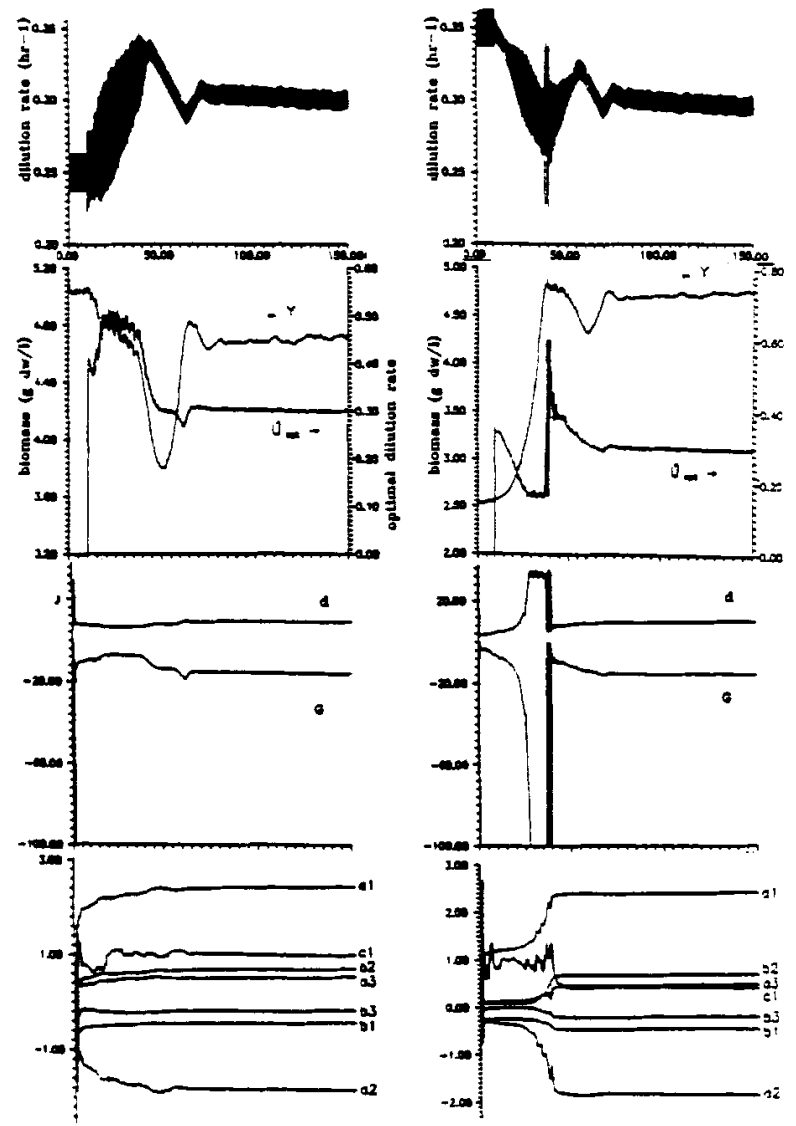

Figure 4: optimizing control transient starting from steady operation at $D=0.25$ $h^{-1}$. a) The perturbed dilution rate.

b) The biomass concentration $y$ and $\delta$

c) Estimates for the static gain $G$ and ${ }^{*}$.

d) ARMAX parameter estimates.

Parameter values ${ }_{-1} T_{\text {opt }}=0.2 \mathrm{~h}, \mathrm{c}=0, \mathrm{~h}_{\mathrm{a}} \mathrm{C}^{-1}$

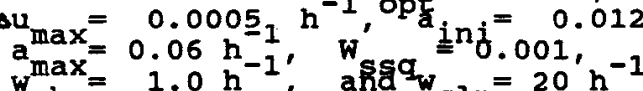

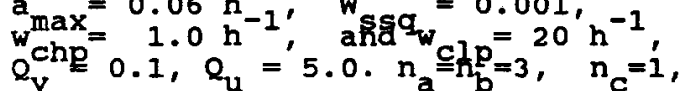

Figure 5: Optimizing control transient starting from steady operation at $\mathrm{D}=0.35$ $\mathrm{h}^{-1}$. Parameters shown in text of Fig. 4 .
With the process operating around a steady state, the two lower levels of the optimizing control hierachy are activated in the following way: First the manipulated variable is perturbed with a pseudo random binary signal, generated by the estimation package, with an amplitude of $a_{\text {inj }}$ After 10 hours, the optmization calculations are initiated every $T$ hour, here the perturbation amplitule is varied according to the distance from the calculated optimum.

Two sets of results are presented in figures 4 and 5, where the optimization is initiated at either side of the optimal dilution rate, which is $0.303 \mathrm{hr}^{-1}$ when the substrate concentration is $S_{0}=$ $10 \mathrm{~g} / \mathrm{l}$. In both cases the optimization strategy is able to bring the process close to the optimal value around 60 hr's after initiation of the optimization. In both cases an oscillatory approach of the dilution rate towards the optimal value is seen in fig.'s $4 \mathrm{a}$ and 5a. The oscillation is followed by a slow transient approach towards the optimal value. A closer inspection of the estimated gain and constant term, reveals however significant differences between the two optimizations. In figs. $4 \mathrm{a}-\mathrm{d}$ the optimum is first overestimated significantly, as seen in fig. $4 \mathrm{~b}$. While the dilution rate is being increased, the optimum is evaluated close to the correct value around 50 hours, and the pertubations are reduced to their minimum, and the dilution rate is decreased. While the dilution rate is 6-128 above the optimal value the static process gain is very low, as seen on fig. $1 b$. This low gain is probably why the static gain estimate reaches a minimum around 60 h. From this point on the estimates behaves very smoothly. Note that the overshoot in dilution rate between 40 and $50 \mathrm{~h}$, results in an undershoot in biomass.

When the optimization is started from above, the process has to pass through the region with very low static gain as seen in fig. 1b. In fig. $5 c$ it is clearly seen that the estimation is able to detect the low static gain, with some delay though. When the gain estimate starts to deviate from the lower limit of -100 , it is seen to be rather uncertain and oscillates between small negative values and the lower limit just before $40 \mathrm{~h}$. This uncertainty is also seen in the calculated optimum. These variations give rise to perturbations which are close to the maximal value. The parameter estimates and the static gain and constant term estimates thereafter converge smoothly as the process approaches the optimum.

With the chosen strategy the above results illustrate that while the process 
is relatively far from the optimum, the approach towards the optimum is limited by the maximally allowed step size in the optimization. The oscillatory approach towards optimum is caused by the delay in estimation of the process gain. The maximally allowed optimization step size may be reduced with resulting decreasing amplitude of the oscillations, this will naturally also slow down the approach towards optimum.

In the above simulations the model order was selected to $n_{a}=n_{b}=3$. This model order renders it possible to perform the optimization both from above and below the optimal dilution rate. Lower model orders does not converge from above. A model order of three is similar to the result obtained by Andersen and Jørgensen (1988) concerning identification of fermentation dynamics on the same simulation model, using multiple measurements.

The effect of using the stabilizing regulator is illustrated in figures 6-8. In Figure 6 the optimization is started from above the optimal dilution rate, as in Fig. 5, only now $Q_{y}=0$; thus the stabilizing regulation ${ }^{\prime s}$ deactivated. The effect hereof is clearly seen in a doubling of the duration of the oscillatory part of the approach to the optimal value. The medium frequency disturbance rejection ability of the stabilizing regulator thus is very beneficial for the optimizing control. In pigure 7 and 8 a one hour pulse disturbance in the substrate concentration from 10 to 20 $\mathrm{g} / \mathrm{l}$ is introduced at $t=70 \mathrm{~h}$. The stabilizing controller is active in Figure 7 , but not in Figure 8 . The effect of the stabilizing controller is clearly seen in that after an initial transient period of around $50 \mathrm{~h}$, the fermentor is stabilized near the optimal value in Fig. 7 . In contrast in Fig. 8 it is difficult for $u$ to recover from the lower bound, andefnly around $150 \mathrm{~h}$ after the disturbance is the fermentor approaching the optimal operating point.

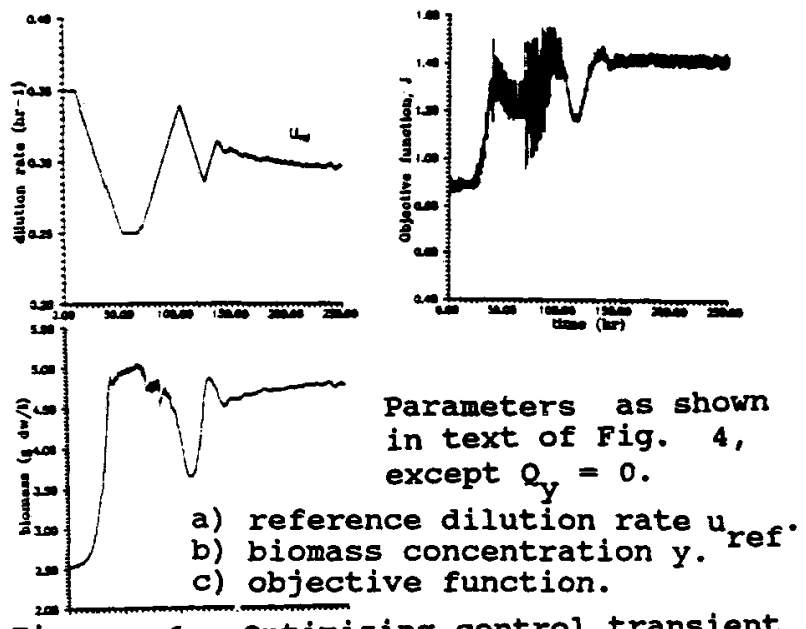

Figure 6: optimizing control transient starting from steady operation at $D=0.35 \mathrm{~h}^{-1}$
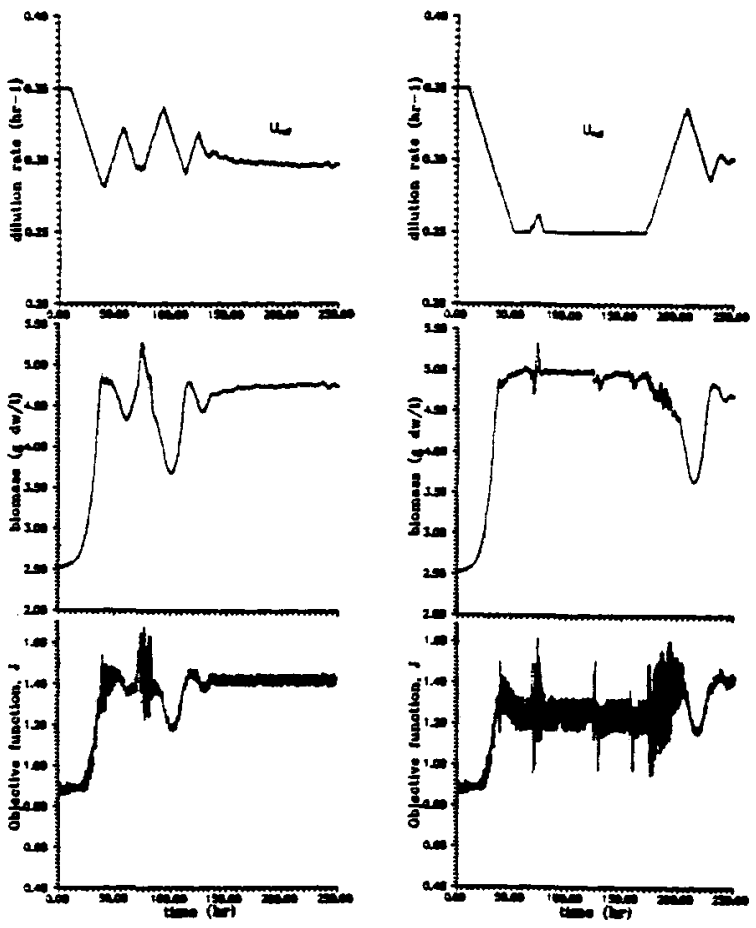

Figure 7-8: Optimizing control transient starting from steady operation at $D=0.35$ $\mathrm{h}^{-1}$. A $10 \mathrm{~g} / \mathrm{l}$ pulse disturbance of $1 \mathrm{~h}$ duration is introduced at $70 \mathrm{~h}$ in substrate concentration $S_{0}$.

curves as in Fig. 6. Parameters as shown in text of Fig. 4, except Fig. 8 where $Q_{y}=0$.

The ability to adapt to constant changes in the substrate concentration is demonstrated in Fig. 9, where a doubling of the substrate concentration occurs at $t=$ $100 \mathrm{~h}$. The objective function reaches the neighbourhood of the optimum relatively fast, some $30 \mathrm{~h}$ after the step change.

The sensitivity towards measurement noise, depends upon the applied filter corner frequencies and the value of the expected standard deviation of the noise, ie. $W_{\text {. }}$. In Figure 10 the effect of a $10 \mathrm{mg} / \mathrm{PS}$ ieasurement noise on the biomass measurement is illustrated. With the increased noise level the parameter estimates becones more noisy as well, this also affect the estimates of the static gain and the constant term, these values in turn affect the determination of $u_{\text {fef' }}$ which is clearly more oscillatory'8f' fig. I0a, than on fig. 4b. The effect of the noise, appart from the obviously increased noise level, is a slightly increased response time. The optimum is determined reasonably well both after the initiation of the transient and after the doubling of the substrate concentration. 

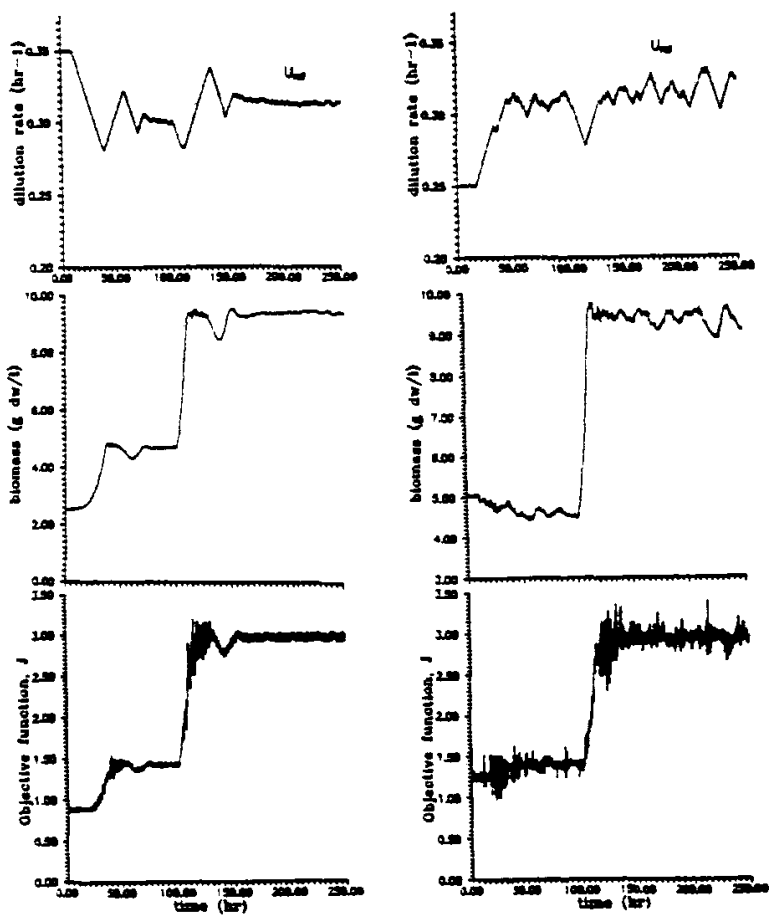

Figure 9: Optimizing control transient starting from steady operation at $D=0.35$ $\mathrm{h}^{-1}$. A $10 \mathrm{~g} / \mathrm{l}$ step increase in substrate concentration $s$ is introduced at $100 \mathrm{~h}$. Curves as in Fig.6. Parameters as shown in text of Fig. 4.

Figure 10: Optimizing control transient starting from steady operation at $D=0.25$ $\mathrm{h}^{-1}$. A biomass measurement noise with 10 $\mathrm{mg} / \mathrm{l}$ standard deviation is introduced. A $10 \mathrm{~g} / 1$ step increase in substrate concentration $S$ is introduced at $100 \mathrm{~h}$. curves as in Fig.6. Parameters as shown in text of Fig. 4 .

\section{CONCLUSIONS}

The behavour of optimizing control on a simulated yeast fermentation is investigated over a practically reasonable operating range. Despite large variations in process gain it is possible to achieve convergence to the optimal value of the objetive function within a couple of open loop response times. The behaviour of the optimizing algoritm

towards medium and low frequency disturbances in substrate concentration is most satisfactory. The algorithm also performs satisfactorily in the presence of measurement noise.

The applied optimizing strategy consists of a three level structure with a lower level containing a stabilizing regulator. This stabilizing regulator rejects medium and high frequency disturbances and thereby enables the optimizing controller to reach the optimal level faster than without the regulating effect.

Figure 3: Three level optimizing control hierachy. IP is a fourth order Butterworth lowpass filter. Hp is a first order high pass filter (eq.4).

\section{REFERENCES}

1. Andersen, M.Y.; S.B.Jørgensen (1988): Identification of a simulated yeast fermentation. Fourth Int. Cong. Comp. Ferm. Processes. Cambridge UK.

2. Bhattacharya, A; B. Joseph (1982): On-line Optimization of Chemical Processes. Proc. ACCp334

3. Brabrand, H. ; S.B.Jørgensen (1988): Robust Multivariable Adaptive Process Control. IFAC workshop on Robust adaptive Control.Newcastle Australia.

4. Chang, Y.K. ; Pyun, Y.R.; Lim,H.C. (1988): Adaptive optimization of a continuous culture with the use of a bilevel Forgetting Factor. Biotech. Bioeng. 31 p 944-957.

5. Garcia,C; Morari, M. (1981 and 1984): optimal operation of Integrated processing systems. Part I AIChE J 27p960; and Part II. ibid 30 p226

6. Golden, M.; E.Ydstie (1987): Nonlinear Adaptive Optimization of Continuous Bioreactors. Proc. IFAC world Conf. Munich p356.

7. Harmon, J; S.Svornos and G.Lyberatos (1985): Adaptive Optimization of $\mathrm{Mi}$ crobial Growth Processes. Proceedings ACC p652.

8. Harmon, J; S.Svornos and G.Lyberatos (1987): Adaptive Steady state optimization of Biomass productivity in Continuous Fermentors. Biotech. and Bioeng. 30 p335

9. Jang, $S_{i}$ B.Joseph; H.Mukai:On-line Optimization of Constrained Multivariable Chemical processes. AIChE J 33 p26

10. Iievense, J. (1984). Ph.D. thesis Purdue University.

11.Ljung, L. (1987): Systems Identification - Theory for the User. PrenticeHall

12. Roberts, P; T.Filliams (1981): An Algorithm for Combined system optimization and Parameter Estimation. Automatica 17 p 199

13. Rolf,M.J.; Lim,H.C. (1984): Adaptive on-Line optimization for continuous Bioreactors. Chem. Eng. Commun. 29 p.229-255.

14.Rolf,M.J.; Lim,H.C. (1985): Exprimental Adaptive on-Line optimization of Cellular Productivity of a continuous Bakers' Yeast Culture. Biotech.Bioeng. 27 p.1236-45.

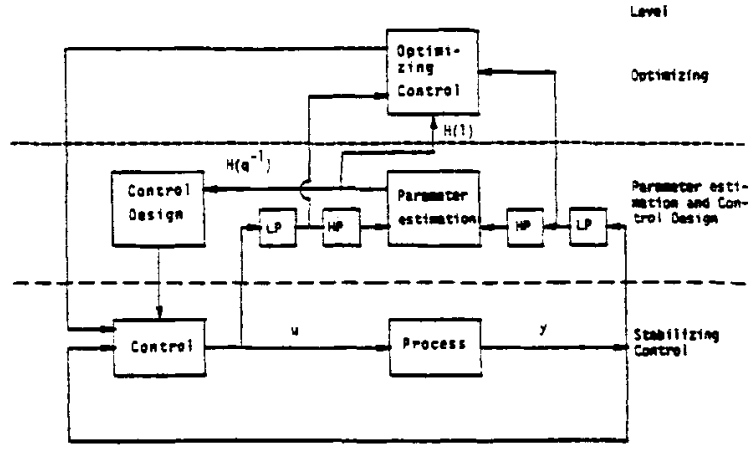

